\title{
Spherical montmorillonite-supported molybdenum disulfide nanosheets as a self-sedimentary catalyst for organic pollutants removal
}

\author{
Hongzhen Wang ${ }^{\mathrm{a}}$, Ning Wang ${ }^{\mathrm{b}, \mathrm{d}, *}$, Fushuai Wang ${ }^{\mathrm{e}}$, Fengyan Xiao ${ }^{\mathrm{b}}$, Dawei Pan ${ }^{\mathrm{b}, \mathrm{c}, \mathrm{d}, *}$ \\ ${ }^{a}$ Qingdao University of Science and Technology, Qingdao 266001, PR China \\ ${ }^{\mathrm{b}}$ CAS Key Laboratory of Coastal Environmental Processes and Ecological Remediation, Shandong Key Laboratory of Coastal Environmental Processes, Research Center for \\ Coastal Environmental Engineering and Technology of Shandong Province, Yantai Institute of Coastal Zone Research, Chinese Academy of Sciences, Yantai 264003, PR \\ China \\ ${ }^{\mathrm{c}}$ University of Chinese Academy of Sciences, Beijing 100049, PR China \\ ${ }^{\mathrm{d}}$ Center for Ocean Mega-Science, Chinese Academy of Sciences, 7 Nanhai Road, Qingdao 266071, PR China \\ e Yantai University, Yantai, Shandong Province 264005, PR China
}

\section{A R T I C L E I N F O}

\section{Keywords:}

Spherical montmorillonite

$\mathrm{MoS}_{2}$

Catalyst

Organic pollutants

\begin{abstract}
A B S T R A C T
Water containing organic and carcinogenic pollutants has become a serious environmental problem, threatening the life of the aquatic ecosystem and human beings. Molybdenum disulfide $\left(\mathrm{MoS}_{2}\right)$, especially monolayered $\mathrm{MoS}_{2}$ sheets, has been proven as an effective catalyst for degradation of organic contaminants due to its more exposed edges and active sites for electron transfer. However, monolayered $\mathrm{MoS}_{2}$ nanosheets easily aggregate due to their high surface energy and strong $\pi-\pi$ electron interaction, heavily affecting the catalytic performances. In addition, monolayered $\mathrm{MoS}_{2}$ nanosheets with small tiny size are difficult to recover from the mixture, restricting the capacity of recyclability and further mass application. Herein, we solved these issues via supporting the small tiny $\mathrm{MoS}_{2}$ nanosheets on spherical montmorillonite (SMt). The SMt can not only provide large pore volume and specific surface area for supporting abundant $\mathrm{MoS}_{2}$ but also reduce the fouling and enhance the transport during the mass production process. The prepared microspheres exhibited a high catalytic activity towards organic pollutants including methylene blue (MB) and 4-nitrophenol (4-NP) in the presence of $\mathrm{NaBH}_{4}$ due to the strong adsorption capacity of SMt and the large catalytic surface area for electron transfer. Furthermore, the micro-sized granular catalyst can be facile recovered and reused without any devices involved due to the excellent self-sedimentary capacity and large size. Moreover, the catalytic performance and the morphology were almost unaltered after recycling 20 times. Our straightforward strategy to solve the issues through porous micro-sized self-sedimentary SMt supporting tiny monolayered $\mathrm{MoS}_{2}$ nanosheet with high catalytic activity facilitates the practical application of these kinds of catalyst towards the reduction of organic and carcinogenic pollutants.
\end{abstract}

\section{Introduction}

Most of the organic dyes and their intermediates are carcinogenic and easily soluble in water [1]. Very low concentration in water can alter the color, thus changing the refraction index of water, which is not only caused the visual pollution but also interfered with the absorb sunlight of aquatic life [2-5]. To solve this problem, a variety of approaches have been developed to remove these pollutants, including physical adsorption [6,7], chemical and biological methods [8]. Among them, chemical catalytic method has been proved as an effective approach to fast degradation with low cost and easy operation [9-14]. Layered ultrathin two-dimensional (2D) nanomaterials have become one of the most promising catalytic materials due to their capacity of providing abundant active sites for catalysis $[15,16]$. As one of the typical 2D nanomaterials, molybdenum disulfide $\left(\mathrm{MoS}_{2}\right)$ nanosheets consist of covalently bonded S-Mo-S layers [17]. Compared with bulk $\mathrm{MoS}_{2}$, mono- or few layered $\mathrm{MoS}_{2}$ nanosheets provide abundant active sites for catalytic reduction of organic pollutants due to its under-coordinated sulfur atoms at the edges [18]. However, the nanosheets easily stack together due to van der Waals interaction during the

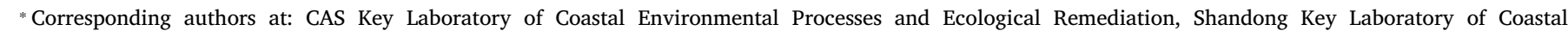

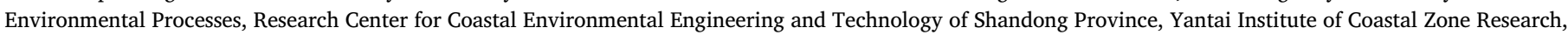
Chinese Academy of Sciences, Yantai 264003, PR China.

E-mail addresses: nwang@yic.ac.cn (N. Wang), dwpan@yic.ac.cn (D. Pan). 
catalytic process, thereby heavily decrease the number of active sites and catalytic activity. Previously, nanoparticles such as carbon nanotube [19], graphene [20,21], and $\mathrm{TiO}_{2}$ [22] were used as the nanosupported substrate to disperse and anchor the $\mathrm{MoS}_{2}$ nanosheets. These nano-sized substrates were well hybridized or wrapped by the $\mathrm{MoS}_{2}$ nanosheets to obtain the stable carbon nanotube/ $\mathrm{MoS}_{2}$, graphene/ $\mathrm{MoS}_{2}$, and $\mathrm{TiO}_{2} / \mathrm{MoS}_{2}$ nanocomposites. Nevertheless, the application of these fabricated nanocomposites was still limited by the critical issues of recyclability due to their tiny nano size.

Montmorillonite (Mt) is a natural nanomaterial, has strong adsorption capacity and has been used as a good substrate for supporting $\mathrm{MoS}_{2}$ [23-29]. For example, Peng et al. designed and successfully fabricated Mt/MoS 2 catalyst with $\mathrm{MoS}_{2}$ nanosheets intercalating in the interlayer of Mt [30] and $\mathrm{MoS}_{2}$ nanosheets depositing on the surface of Mt [31]. Although the prepared Mt/MoS 2 catalyst exhibited a good catalytic activity towards organic pollutant 4-nitrophenol (4-NP), their mass applications are still restricted by the problem of recyclability because the deposit rate of Mt based catalyst in the mixture is highly limited by the excellent hydrophilicity and swelling ability of Mt. Therefore, a great number of recovered devices are involved which heavily increases the cost of wastewater treatment.

Spray-drying is a proven technique in the industry to fabricate granulation with microsize to millimeter-size [32]. In our previous work, Mt were granulated to spherical micro-sized microsphere through this technology [33-35]. The stacked Mt forms a porous structure and provides abundant surface areas to support active sites. In addition, the granular treatment can obviously enhance the transport ability during the productive process, facilitating mass prodution of these kinds of catalysts [36]. Furthermore, the density of the prepared granular Mt microsphere is much higher than the mixture, which can be self-sedimentary and easy recovered from the bottom. In this work, we fabricated micro-sized spherical Mt (SMt) with large specific surface areas, followed by adhering to the tiny $\mathrm{MoS}_{2}$ nanosheets on the surface of SMt through the polydopamine (PDA) coating [37]. PDA has been demonstrated the strong adhesion on various substrates [38]. The model organic pollutants, methylene blue (MB) and 4-nitrophenol (4-NP) were selected to evaluate the catalytic performances. Moreover, the recyclability was investigated. As we know, this is the first report of synthesized SMt/MoS ${ }_{2}$ for catalytic reduction of MB and 4-NP, successfully solving the engineering problem through a physical method.

\section{Experimental}

\subsection{Chemicals}

All chemicals and reagents were used as received without any further purification. They include dopamine hydrochloride (DA, Sigma Aldrich, $\geq 98.0 \%$ ), monolayered molybdenum disulfide $\left(\mathrm{MoS}_{2}\right.$, SigmaAldrich, $\geq 98.0 \%$ ), montmorillonite (Mt, (Na, K, Ca) ${ }_{0.33}\left(\mathrm{Al}_{1.67} \mathrm{Mg}_{0.33}\right)$ $\mathrm{Si}_{4} \mathrm{O}_{10}(\mathrm{OH})_{2} \cdot \mathrm{nH}_{2} \mathrm{O}$ ), Sinopharm Chemical Reagent Co. Ltd., China, $\geq 98.0 \%$ ), methylene blue (MB, Tianjin Guangfu Reagent Co., China, $\geq 98.0 \%$ ), 4-nitrophenol (4-NP, Tianjin Guangfu Reagent Co., China, $\geq 98.0 \%)$, sodium borohydride $\left(\mathrm{NaBH}_{4}\right.$, Sinopharm Chemical Reagent Co. Ltd., China, $\geq 99.0 \%$ ), and Tris-HCl buffer (Beijing Solarbio Science \& Technology Co., Ltd, China).

\subsection{Granulation of Mt and fabrication of spherical Mt supported $\mathrm{MoS}_{2}$}

Spray drying technology was applied to fabricate spherical Mt (SMt). First, $10.0 \mathrm{~g}$ Mt was mixed with $100 \mathrm{~mL}$ ethanol and sonicated for $4 \mathrm{~h}$. Then the suspension was adding to the spray-dryer (YC-015, Shanghai Pilotech Instrument \& Equipment Co. Ltd) using a peristaltic pump. The feed rate was set as $50 \mathrm{~mL} \mathrm{~min}^{-1}$ and the drying temperature was set as $110{ }^{\circ} \mathrm{C}$, respectively. Finally, the granular SMt with the mean size of about $20 \mu \mathrm{m}$ was obtained. The prepared SMt was used as the substrate to support $\mathrm{MoS}_{2}$. Typically, $1.0 \mathrm{~g}$ DA and $20 \mathrm{mM}$
Tris-HCl buffer were added in $200 \mathrm{~mL}$ water to form the mixture. The $\mathrm{pH}$ was adjusted to 8.5 using $0.1 \mathrm{M}$ of $\mathrm{NaOH}$ solution. After the color of the solution changed to dark yellow, $2.0 \mathrm{~g}$ SMt was added to form the suspension. Through $6 \mathrm{~h}$ shaking, the color of suspension changed to black and then filtered. The black product was fully washed by a $25 \%$ isopropyl alcohol-water solution and then dried in a vacuum oven at $60{ }^{\circ} \mathrm{C}$ for $24 \mathrm{~h}$ to obtained the black microsphere. On the other hand, the monolayered $\mathrm{MoS}_{2}$ nanosheets were mixed in aqueous solution with a concentration of $0.1 \mathrm{mg} \cdot \mathrm{mL}^{-1}$, followed by ultrasound treatment for at least $4 \mathrm{~h}$ via a cell disruptor to get the dark black suspension. The dark black suspension was separated by the centrifugal separation at $12,000 \mathrm{rpm}$ for $2 \mathrm{~min}$. The faint yellow supernatant was extracted and mixed with the prepared black microsphere using a rotator for at least $6 \mathrm{~h}$. After the filter separation, the microspheres were dried to obtain the final product.

\subsection{Characterization}

The Fourier transform infrared (FTIR) spectra of samples were obtained through a Perkin-Elmer 2000 Fourier transform infrared spectrometer with $\mathrm{KBr}$ pressed pellets. Scanning electron microscopy (SEM) was performed using a Jeol S4800 scanning electron microscope and an energy-dispersive detector. Transmission electron microscopy (TEM) and atomic force microscopy (AFM) were carried out using a JEM-2200 transmission electron microscope and Multi-Mode 8 atomic force microscope, respectively. X-ray diffraction (XRD) measurements were performed with Bruker D8-Advantage powder diffractometer using $\mathrm{Cu}$ $\mathrm{K} \alpha$ radiation $(40 \mathrm{kV}, 110 \mathrm{~mA})$. X-ray photoelectron spectroscopy (XPS) measurements were taken using Thermo EscaLab 250Xi spectrometer. Nitrogen adsorption measurements were carried out at $77 \mathrm{~K}$ using an ASAP2020 analyzer. The UV absorption spectra were measured using a PERSEE UT 1810 UV-vis spectrometer.

\subsection{Adsorption experiment}

$10.0 \mathrm{mg}$ samples were engaged in a $100 \mathrm{~mL}$ aqueous solution containing $100 \mathrm{mg} \mathrm{L}^{-1} \mathrm{MB}$ and fully mixed. Then the UV-vis absorption spectra of organic pollutants at a certain interval were recorded. The removal percentages of organic pollutants are calculated following the equation (1) [6]:

$\mathrm{R}=\left(\frac{\mathrm{C}_{0}-\mathrm{C}_{\mathrm{t}}}{\mathrm{C}_{0}}\right) \times 100 \%$

Where $\mathrm{R}$ is the removal percentage of organic dyes, the $\mathrm{C}_{\mathrm{o}}\left(\mathrm{mg} \mathrm{L}^{-1}\right)$ and $\mathrm{C}_{\mathrm{t}}\left(\mathrm{mg} \mathrm{L}^{-1}\right)$ are the initial dye concentration and the concentration of reaction time, respectively.

The equilibrium concentration is calculated according to equation (2) [39]:

$\mathrm{q}_{\mathrm{e}}=\left(\mathrm{C}_{0}-\mathrm{C}_{\mathrm{e}}\right) \mathrm{V} / \mathrm{W}$

Where $\mathrm{q}_{\mathrm{e}}$ is the amounts of organic pollutants adsorbed on the samples at equilibrium, $\mathrm{C}_{0}\left(\mathrm{mg} \mathrm{L}^{-1}\right)$ and $\mathrm{C}_{\mathrm{e}}\left(\mathrm{mg} \mathrm{L}^{-1}\right)$ are the initial and equilibrium concentrations of organic pollutants solution, respectively, $\mathrm{V}$ is the volume of the reaction solution (L) and $\mathrm{W}$ is the mass $(\mathrm{g})$ of the samples.

The reduction efficiency of organic pollutants is calculated following the equation (3):

$E=\left(\frac{C_{0}-C_{t}}{C_{0}}\right) \times 100 \%$

Where $\mathrm{E}$ is the reduction efficiency of organic pollutants, the $\mathrm{C}_{\mathrm{o}}$ (mg $\left.\mathrm{L}^{-1}\right)$ and $\mathrm{C}_{\mathrm{t}}\left(\mathrm{mg} \mathrm{L}^{-1}\right)$ are the initial and equilibrium concentration of organic pollutants, respectively. 


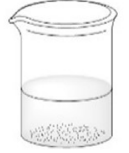

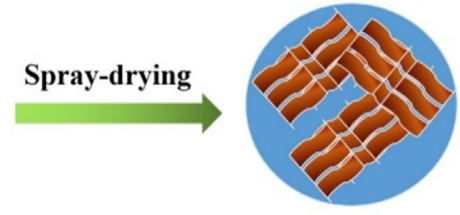

Granular Mt (SMt)

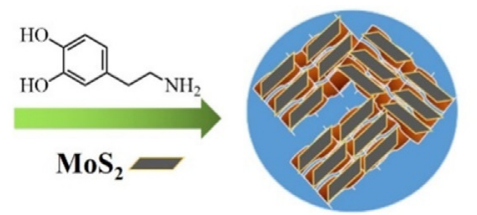

SMt/MoS 2 Microspheres
Scheme 1. The synthesized route for the fabrication of $\mathrm{SMt} / \mathrm{MoS}_{2}$ microsphere, including the first spray-drying and further fixed tiny $\mathrm{MoS}_{2}$ nanosheets on the prepared SMt through dopamine chemistry method.

\section{Mt/Ethanol Suspension}

\subsection{Catalytic reduction}

The catalytic reduction of organic dyes was carried out to assess the catalytic activity and reusability of the prepared microsphere. The calibration curves of organic dyes and their intermediates are shown in Fig. S1. Typically, $10 \mathrm{~mL}$ organic dyes $\left(250 \mathrm{mg} \mathrm{L}^{-1}\right)$ and $20 \mathrm{mg}$ $\left(200 \mathrm{mg} \mathrm{L}^{-1}\right.$ ) catalyst were mixed with $80 \mathrm{~mL}$ deionized water. After fully stirring, $10 \mathrm{~mL} \mathrm{NaBH}_{4}(0.5 \mathrm{M})$ aqueous solutions was added. As the color of organic dyes vanished, the prepared microsphere was easily separated from the mixture due to the self-sedimentary property. Then, the catalyst was further added to the new solution containing organic dyes and $\mathrm{NaBH}_{4}$ to finish one cycle. All the processes were fully recorded by the UV-vis spectrometer.

\section{Results and discussion}

\subsection{Synthesis and characterization of SMt/MoS 2 microspheres}

Porous granular Mt substrates were first prepared through the spray-drying technology. As shown in Scheme 1, after fully sonicated with ethanol, the mixed suspension was granulated through a spray dryer. The granular Mt was modified by dopamine and a black coating was formed on the surface. The strong adhesion capacity of PDA coating could easily fix the tiny $\mathrm{MoS}_{2}$ nanosheets and thus fabricated the SMt/ $\mathrm{MoS}_{2}$ microsphere.

The granulation process of Mt is not only enhanced the size of Mt but also improved the pore size and flowability of the prepared catalyst [36]. Therefore, the morphology was observed by the SEM, TEM, and AFM. As shown in Fig. 1a, the pristine Mt exhibits an amorphous morphology with the Mt sheets loose stacking (Fig. S2a). After the spray-drying process, the granular particle with a smooth surface and a mean diameter of about $20 \mu \mathrm{m}$ were successfully fabricated (Fig. 1b). The PDA coated process has almost unaltered the morphology (Fig. 1c). However, the obviously rough surface could be observed after deposited $\mathrm{MoS}_{2}$ nanosheets (Fig. 1d). Difference from the prinstine Mt, a dense and porous morphology with Mt sheets tight stacking can be observed from Fig. S2b. As shown in the inset of Fig. 1d and Fig. S3, a thin $\sim 4 \mathrm{~nm}$ layer was well deposited on the SMt. The small nanosheets were determined to be $\mathrm{MoS}_{2}$, exhibiting strong Mo and S element peaks by EDX shown in Fig. S4. The crystal phases of $\mathrm{MoS}_{2}$, SMt, and SMt/ $\mathrm{MoS}_{2}$ microsphere were analyzed by X-ray diffraction, and the data is exhibited in Fig. 2. The SMt with the crystallite size of $14.9 \mathrm{~nm}$ calculated from the (100) peak using Scherrer equation contained smectite (fcc, JCPDS card no. 13-0135) and quartz (fcc, JCPDS card no. 461045) [40]. The peaks at $2 \theta$ of $14.2^{\circ}, 32.9^{\circ}, 39.1^{\circ}, 48.5^{\circ}$, and $58.3^{\circ}$ appear, which can be assigned to the (002), (100), (103), (105), and (110) crystal faces of $2 \mathrm{H}-\mathrm{MoS}_{2}$ (fcc, JCPDS card no. 37-1492), confirming the successfully supported $\mathrm{MoS}_{2}$ on the SMt (Fig. 2) [20,25]. The crystallite size of $\mathrm{MoS}_{2}$ nanosheets was estimated from (002) peak as $7.7 \mathrm{~nm}$, which is similar to the result of AFM (the thickness of the nanosheets is about $4 \mathrm{~nm}$, Fig. 1e). The small-sized nanosheets can be easily supported on the micro-sized SMt substrates and provided abundant active sites for the reaction. For the SMt/MoS 2 microsphere, it exhibits the peaks of SMt and $\mathrm{MoS}_{2}$, reflecting the good composition of $\mathrm{SMt} / \mathrm{MoS}_{2}$ microsphere. The crystallite size of SMt/MoS 2 microsphere was calculated as $14.5 \mathrm{~nm}$, which is almost the same with the SMt, demonstrating the higher content of SMt in the SMt/MoS 2 microsphere. XPS measurements were carried out to investigate the surface elemental composition and chemical status of the prepared microsphere. As shown in Fig. 1f, the peaks corresponding to $\mathrm{C}, \mathrm{O}, \mathrm{Si}, \mathrm{S}, \mathrm{Al}$, and Mo can be observed, well consistent with the formation of SMt/MoS $\mathrm{Micro}_{2}$ sphere. The atomic percentages of Mo and S elements on the surface of the microsphere were calculated as $0.11 \%$ and $0.17 \%$, which is well agreeing with the theoretical values for $\mathrm{MoS}_{2}$. As shown in Fig. S5, for the pristine $M_{0}$ nanosheets, the peaks of Mo $3 d_{3 / 2}$, Mo $3 d_{5 / 2}$, and $S$ $2 \mathrm{~s}$ are $233.1 \mathrm{eV}, 229.7 \mathrm{eV}$, and $226.9 \mathrm{eV}$, respectively. The higher peak at $237.1 \mathrm{eV}$ is assigned to the $\mathrm{Mo}_{3} \mathrm{~d}_{3 / 2}$ of $\mathrm{MoO}_{3}$, which is derived from the adsorbed oxygen molecule [20]. For the samples of SMt/MoS $\mathrm{mi}_{2}$ crosphere, no peak of $\mathrm{MoO}_{3}$ can be observed (Inset of Fig. 1f), demonstrating the no effect of catalytic activity of SMt/MoS $\mathrm{S}_{2}$ microsphere. Furthermore, the peaks of Mo $3 \mathrm{~d}$ are shifted to the higher energy value, reflecting the electronic interaction between $\mathrm{MoS}_{2}$ and SMt. The enhanced interaction facilitates the improve the stability of microsphere, thereby maintaining the catalytic activities and benefiting the recovery and reuse. In addition, corresponding to the TEM image, the EDX mapping of Mo and S elements exhibit the same region, demonstrating the immobilization supporting of $\mathrm{MoS}_{2}$ nanosheet in the fabricated microsphere (Fig. 3). Furthermore, the loading of Mo was quantified by ICP/MS to be $0.4 \mathrm{wt} \%$ upon dissolving SMt $/ \mathrm{MoS}_{2} \mathrm{mi}-$ crosphere in the strong acid, that is, the loading of $\mathrm{MoS}_{2}$ was calculated to be $0.67 \mathrm{wt} \%$. Considered the small tiny size $\mathrm{MoS}_{2}$ uniformly dispersion on the SMt, the content of loading $\mathrm{MoS}_{2}$ nanosheet can provide the abundant tiny active sites for catalytic reduction of organic pollutants.

\subsection{Adsorption experiments}

Through the granulation process, the Mt was stacked to the $2 \mathrm{D}$ microsphere, thus the changes of specific surface area and pore properties were measured by the $\mathrm{N}_{2}$ adsorption. As shown in Fig. $4 \mathrm{a}$ and Table 1, compared with pristine Mt with a BET specific surface area of $8.2 \mathrm{~m}^{2} \mathrm{~g}^{-1}$ and a total pore volume of $0.1 \mathrm{~cm}^{-3} / \mathrm{g}$, the values of granular SMt greatly improved to $60.6 \mathrm{~m}^{2} / \mathrm{g}$ and $0.38 \mathrm{~cm}^{-3} / \mathrm{g}$, respectively, reflecting the well pore-making ability of spray-drying. Furthermore, the values were slightly decreased to $45.6 \mathrm{~m}^{2} / \mathrm{g}$ and $0.24 \mathrm{~cm}^{-3} / \mathrm{g}$ after the surface coating process and further decreased to $31.3 \mathrm{~m}^{2} / \mathrm{g}$ and $0.20 \mathrm{~cm}^{-3} / \mathrm{g}$ after the supporting process, which is still much higher than pristine Mt. The decreased values after the supporting process is mainly due to the weak $\mathrm{N}_{2}$ adsorption capacity of $\mathrm{MoS}_{2}$, as shown in Fig. 4a and Table 1. The large pore size will benefit the adsorption and catalytic reduction of organic dyes due to the opportunity for contacting with the catalyst and organic dyes. The adsorption towards organic dyes was monitored by UV-Vis absorption spectra and MB with the band of $665 \mathrm{~nm}$ was selected as a model here. Samples including pristine Mt, SMt, PDA-coated SMt, and SMt/MoS exhibited similar adsorption with three stages, the first fast adsorption process, gradually increased stage, and the final equilibrium stage. Although all samples exhibited similar first fast adsorption behaviors, the gradual stage is quite different due to the differences of the intra-particle diffusion-rate controlling process. Compared with the pristine Mt, the intra-particle diffusion-rate of SMt, PDA-coated SMt, and SMt/MoS is prolonged. The samples reach the equilibrium stage as the max 

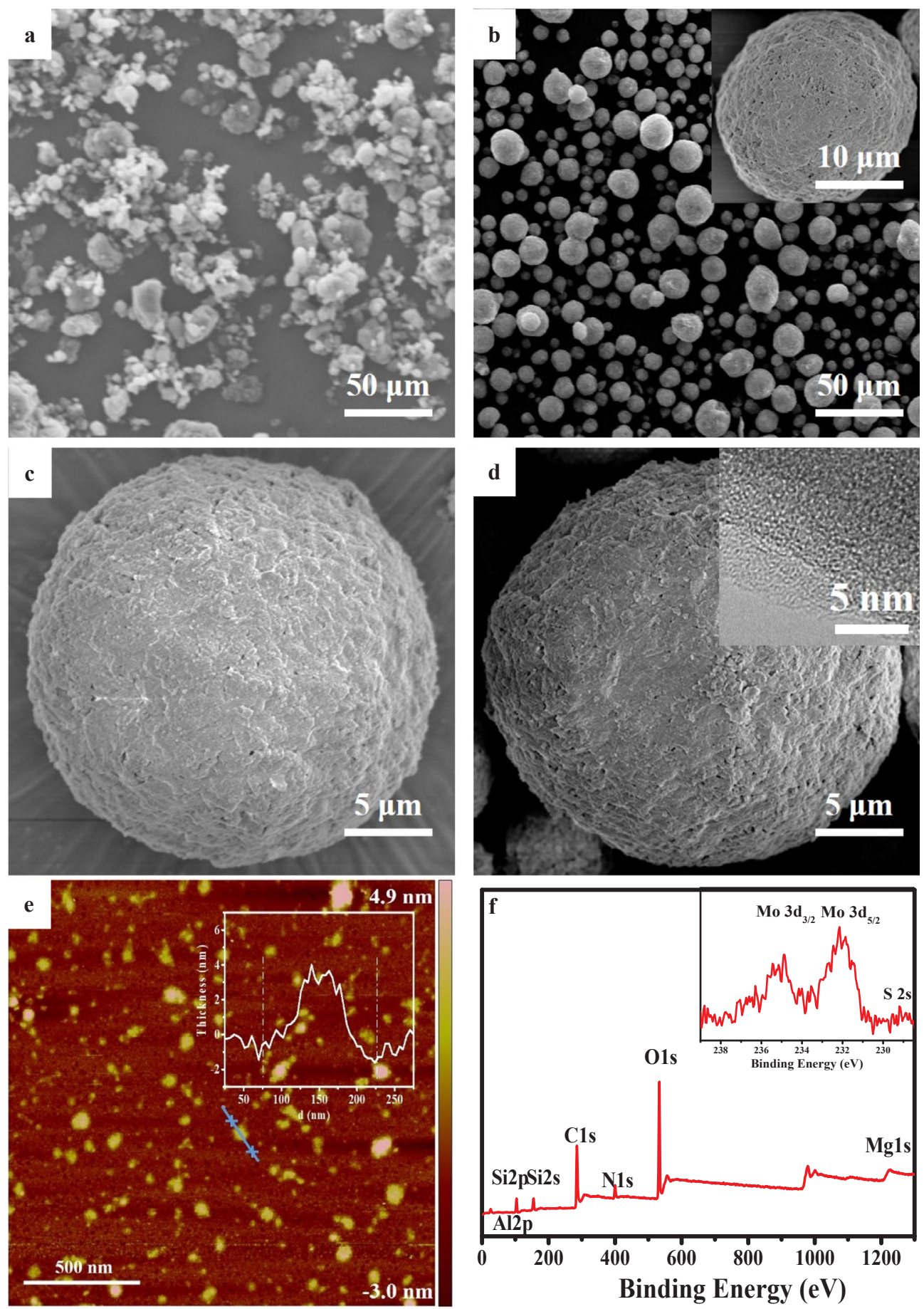

Fig. 1. SEM images of the Mt (a), SMt (b) with inset enlarging image, PDA@SMt (c) and SMt/MoS ${ }_{2}$ (d) microspheres with inset high-resolution TEM spectra. AFM image with inset corresponding thickness of $\mathrm{MoS}_{2}$ nanosheet (e). XPS spectra of SMt/MoS 2 with inset high-resolution XPS spectra of Mo 3d and S 2s regions (f).

adsorption is achieved (Fig. 4b). Among the samples, SMt exhibited the largest adsorption capacity of $992 \mathrm{mg} \mathrm{g}^{-1}$, which is increased about $68.7 \%$ compared with pristine Mt. It can be attributed to the well granular process of Mt. SMt/MoS 2 slightly decreased the adsorption capacity to $870 \mathrm{mg} \mathrm{g}^{-1}$, the value is much higher than other substrates reported in the works of literature (150 $\mathrm{mg} \mathrm{g}^{-1}$ [41], $365 \mathrm{mg} \mathrm{g}^{-1}$ [22]). The enhanced adsorption ability can effectively improve the concentration of organic pollutants near the surface of the catalyst, thus improve the catalytic activity [39]. As the high adsorption capacity was obtained, the high catalytic performance is highly desirable.

\subsection{Catalytic properties}

The catalytic capacity of the prepared SMt/MoS 2 microsphere was evaluated through the color variation of organic dyes suffering from the catalytic reduction under $\mathrm{NaBH}_{4}$. The peaks of MB and 4-NP, with the initial peak at $665 \mathrm{~nm}$ and $400 \mathrm{~nm}$, respectively, were decreased with the prolonging catalytic reduction time. The process can be easily monitored by UV-vis spectroscopy. As shown in Fig. 5a and 5b, the absorbance peak decreases rapidly within $7 \mathrm{~min}$ and $6 \mathrm{~min}$, respectively. The catalytic reduction process is not only eliminated the visual pollution but also alter the carcinogenicity to low and no bioactivity [34]. For example, upon the catalytic reduction process, the color of the 


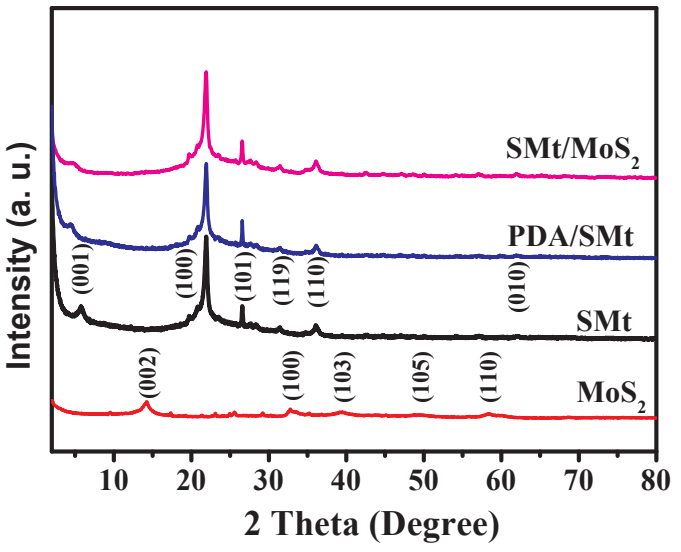

Fig. 2. XRD patterns of SMt, $\mathrm{MoS}_{2}$, PDA/SMt, and SMt/MoS 2 .

MB solution changed from blue to almost colorless, accordingly, the carcinogenic MB was altered to inactive form leucomethylene blue (LMB) [35]. Furthermore, reaction kinetics were investigated. As shown in Fig. $5 c$, both the catalytic processes of samples exhibit the linear relationships, demonstrating the pseudo-first-order reaction kinetics. From the linear relation of $\ln \left(\mathrm{C}_{\mathrm{t}} / \mathrm{C}_{0}\right)$ with the reaction time, the apparent reduction rate constant $(\mathrm{K})$ was obtained. The values of $\mathrm{K}$ were $0.381 \mathrm{~min}^{-1}$ and $0.307 \mathrm{~min}^{-1}$ for MB and 4-NP, respectively. The catalytic mechanism of SMt/MoS ${ }_{2}$ microsphere is based on the electron transfer from the electron donor to the electron accepter [35]. The catalytic activity is mainly related to the surface area providing for electron transfer. Thus, the optimized $\mathrm{K}$ can be obtained through tailoring the concentration of catalyst. As shown in Fig. 5d, the $\mathrm{K}$ values can increase linearly to $0.755 \mathrm{~min}^{-1}$ with the concentration of SMt/ $\mathrm{MoS}_{2}$ microsphere increasing from $200 \mathrm{mg} \mathrm{L}^{-1}$ to $1000 \mathrm{mg} \mathrm{L}^{-1}$ and unaltered concentration of 4-NP and $\mathrm{NaBH}_{4}\left(250 \mathrm{mg} \mathrm{L}^{-1}\right.$ and $0.5 \mathrm{M}$, respectively). The $\mathrm{K}$ towards $4-\mathrm{NP}$ is much higher than the $\mathrm{MoS}_{2}$ $\left(0.235 \mathrm{~min}^{-1}\right)$ and $\mathrm{Mt} / \mathrm{MoS}_{2}\left(0.874 \mathrm{~min}^{-1}\right.$ and $\left.0.723 \mathrm{~min}^{-1}\right)$ reported in the literature due to the good dispersion of $\mathrm{MoS}_{2}$ nanosheets and high adsorption of SMt substrates towards organic dyes [30,31].

\subsection{Recyclability examination}

Considered the cost of $\mathrm{MoS}_{2}$ and water containing organic pollutants, recyclability is the most promising approach for the application of the prepared SMt/MoS 2 microsphere. In this work, successive 20 cycles were applied to evaluate the recyclability of prepared catalysts. As shown in Fig. 6a and b, SMt/MoS 2 exhibits excellent catalytic stability achieving almost $100 \%$ efficiency in the 15 cycles and slightly decreasing less than $1.5 \%$ in the 20 cycles. Furthermore, as shown in Fig. $6 \mathrm{c}$ and d, the catalytic performance is almost unaltered upon 20 cycles. The $\mathrm{K}$ decreased by less than $5 \%$. The mass loss percent of SMt/ $\mathrm{MoS}_{2}$ microsphere after recovery was weighted. After the centrifugation process, the values of the microspheres after $1,5,10$, and 20 cycles for catalytic reduction of $\mathrm{MB}$ were decreased by $98.5 \mathrm{wt} \%, 97.2 \mathrm{wt} \%$, $96.4 \mathrm{wt} \%$, and $95.5 \mathrm{wt} \%$, well agreeing with the decreased ratio of $\mathrm{K}$. Thus, the slightly decreased $\mathrm{K}$ after recyclability experiment can be attributed to the mass loss of catalyst. Furthermore, as shown in Fig. 6e and $\mathrm{f}$, the morphology of the prepared SMt/ $\mathrm{MoS}_{2}$ microsphere was almost unchanged after 20 cycles. In addition, as shown in Fig. S6, the XRD pattern of SMt/MoS $\mathrm{M}_{2}$ microsphere after catalytic cycle 20 times was almost unaltered. All their results reflect the excellent stability of the prepared microsphere.

\section{Conclusions}

The micro-sized granular SMt/MoS $\mathrm{M}_{2}$ microsphere was facile fabricated through the polydopamine method. $\mathrm{MoS}_{2}$ nanosheets stably fixed on the surface of porous SMt, effectively solving the aggregation problem and further providing the abundant active sites. Besides, the prepared microsphere can effectively adsorb the organic pollutants due to its porous structure, thus, improved the concentration of organic pollutants near the surface of catalysts. These advantages validly facilitate the catalytic performance of organic pollutants. Furthermore, the prepared SMt/MoS 2 microsphere can be easily recovered from the mixture without any device involved due to the self-sedimentary property. After recycling 20 times, the morphology of SMt/MoS $\mathrm{Mi}_{2}$ crosphere still keep stable and the catalytic reduction efficiency was almost unaltered. Our straightforward approach to solving the
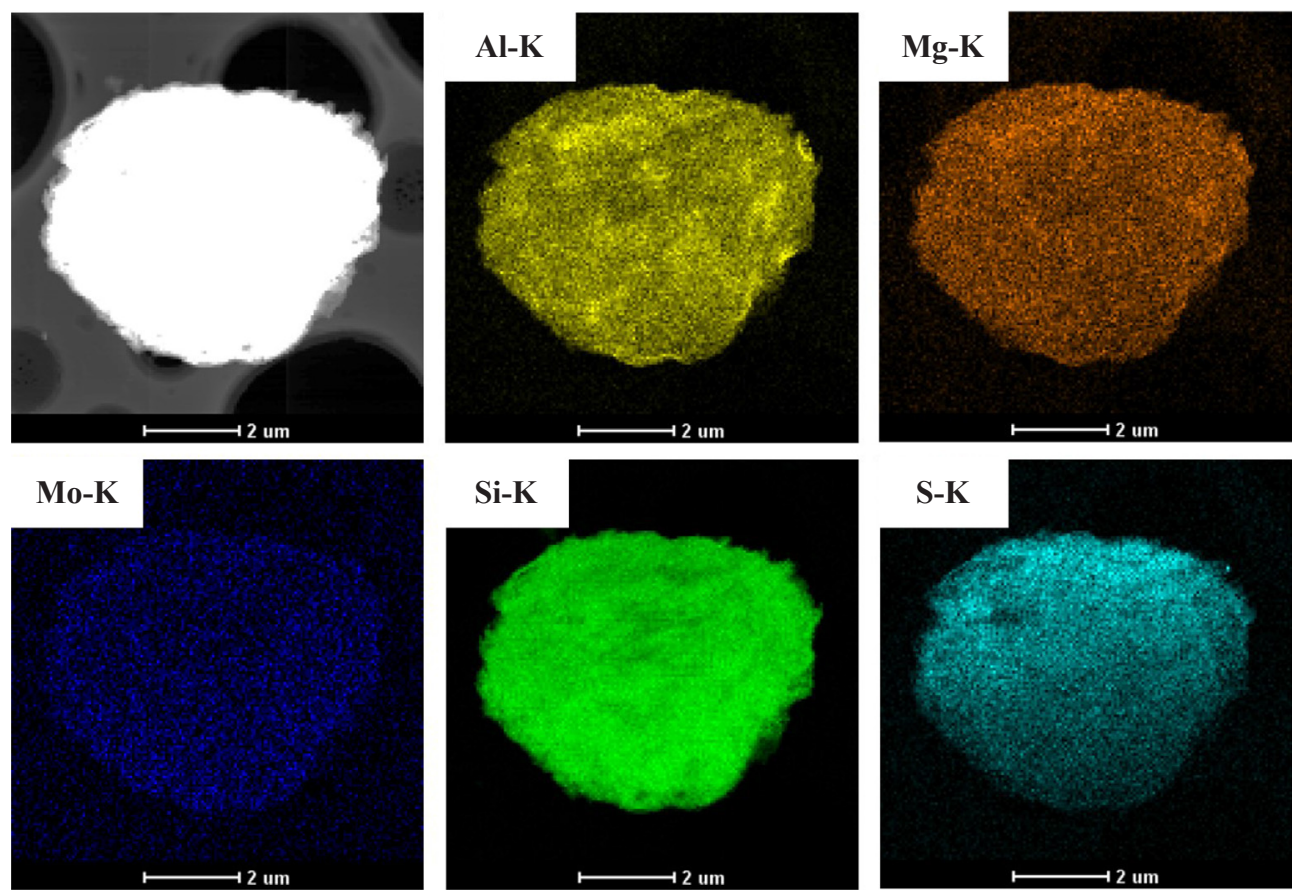

Fig. 3. HAADF-STEM image and the corresponding STEM-EDX elemental mapping images of the SMt/MoS $\mathrm{M}_{2}$ microspheres. 

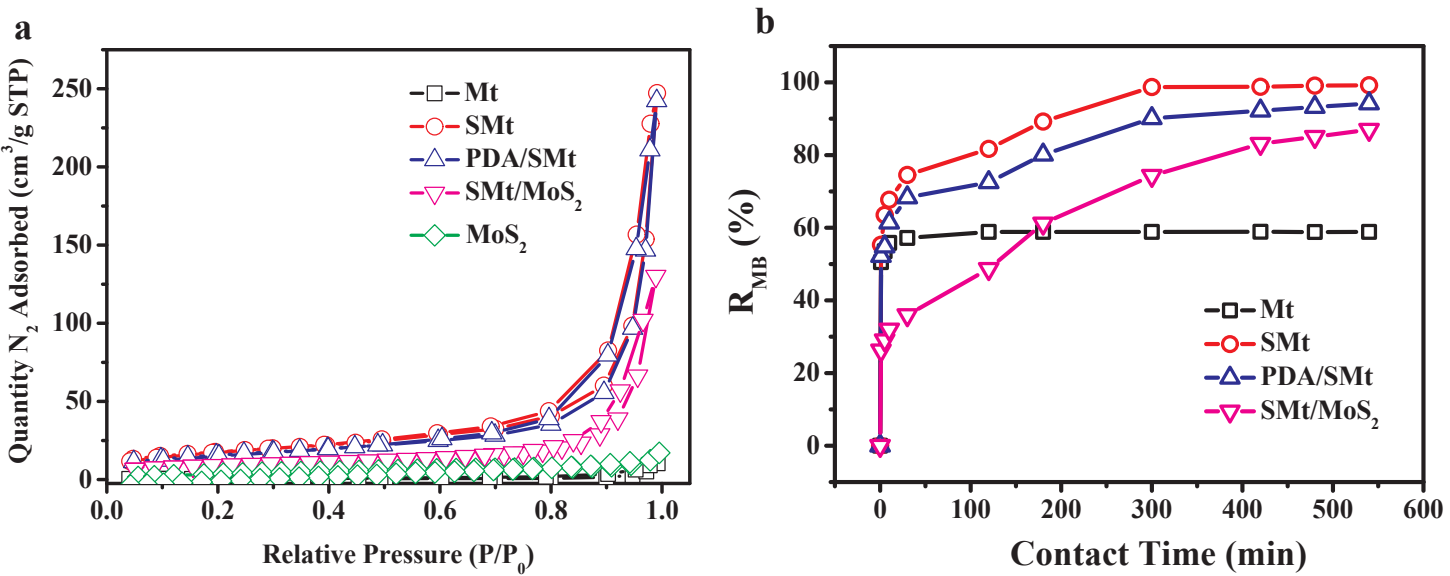

Fig. 4. The $\mathrm{N}_{2}$ adsorption (a) and adsorption kinetics of model organic pollutant MB (b) on the Mt, SMt, PDA/SMt, and SMt/MoS ${ }_{2}$ microspheres.

Table 1

Mesoscale properties of Mt, SMt, PDA/SMt, SMt@MoS 2 , and $\mathrm{MoS}_{2}$.

\begin{tabular}{lll}
\hline Sample & $\begin{array}{l}\text { BET surface area } \\
\left(\mathrm{m}^{2} \mathrm{~g}^{-1}\right)\end{array}$ & $\begin{array}{l}\text { Pore volume } \\
\left(\mathrm{cm}^{3} \mathrm{~g}^{-1}\right)\end{array}$ \\
\hline $\mathrm{Mt}$ & 8.2 & 0.10 \\
$\mathrm{SMt}$ & 60.6 & 0.38 \\
$\mathrm{PDA} / \mathrm{SMt}$ & 45.6 & 0.24 \\
SMt@MoS & 31.3 & 0.20 \\
$\mathrm{MoS}_{2}$ & 16.0 & 0.03 \\
\hline
\end{tabular}

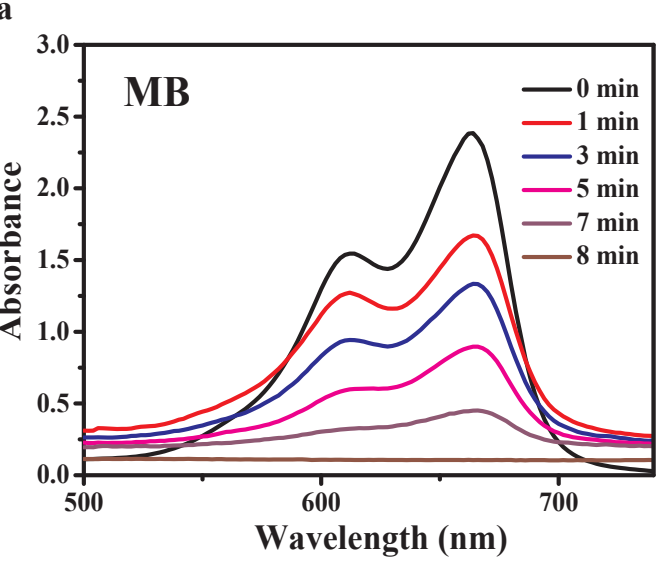

c

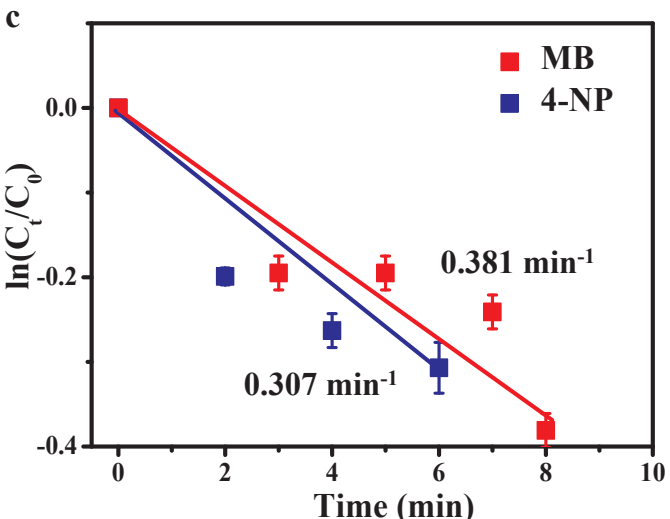

problems of aggregation and recyclability through self-sedimentary micro-sized porous granular nanomaterials as the substrate may pave a promising way to produce high-performance catalyst for application.

\section{Author contributions}

All authors have given approval to the final version of the manuscript.

\section{CRediT authorship contribution statement}

Hongzhen Wang: Writing - original draft. Ning Wang: Writing -

b

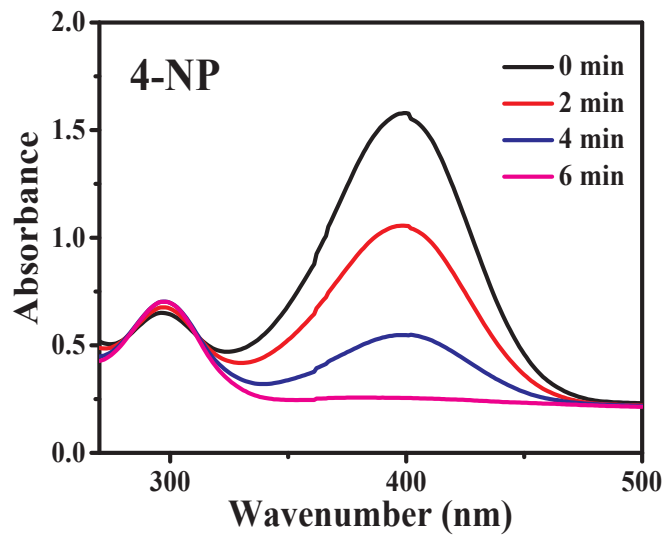

d

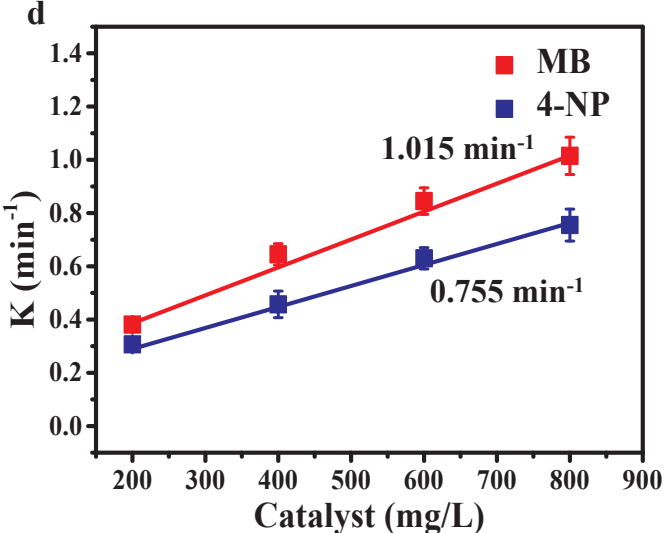

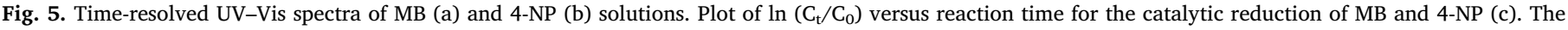
apparent reduction rate constant $(\mathrm{K})$ of $\mathrm{MB}$ and 4-NP varies as a function of the concentration of microspheres (d). 
a

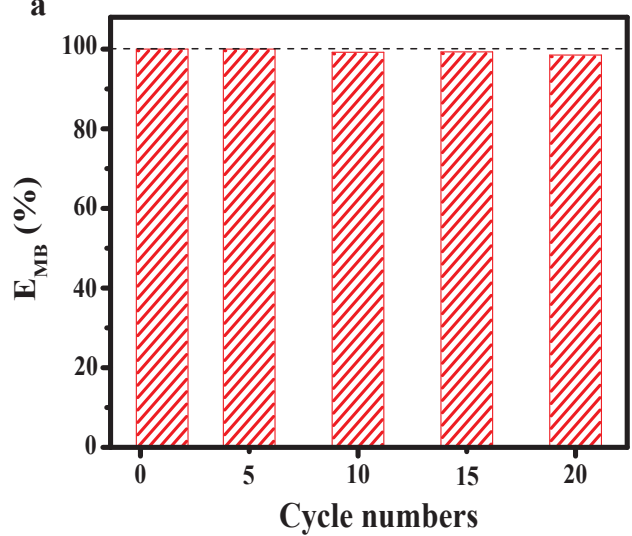

c

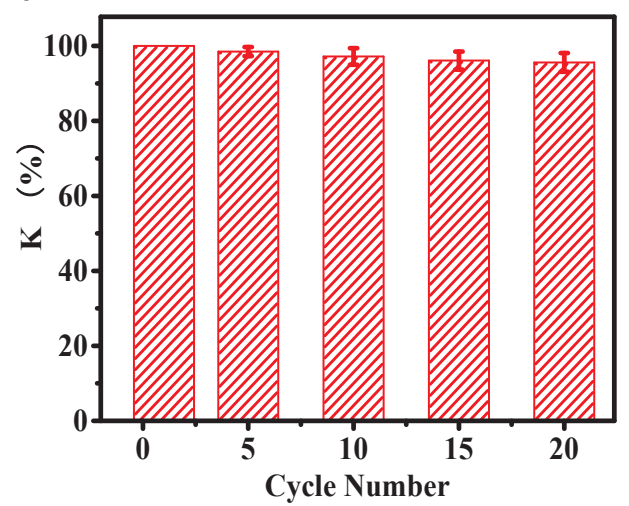

e

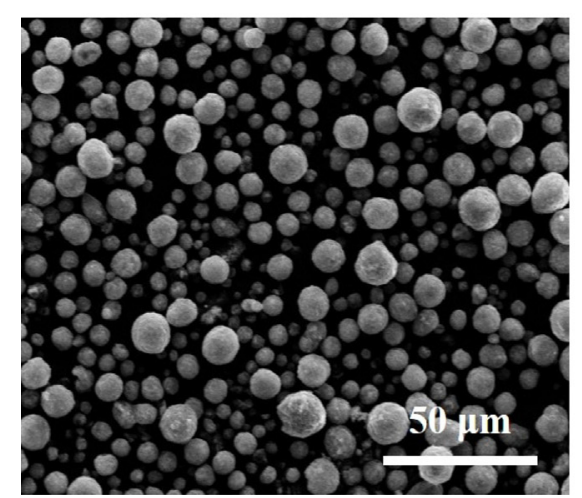

b

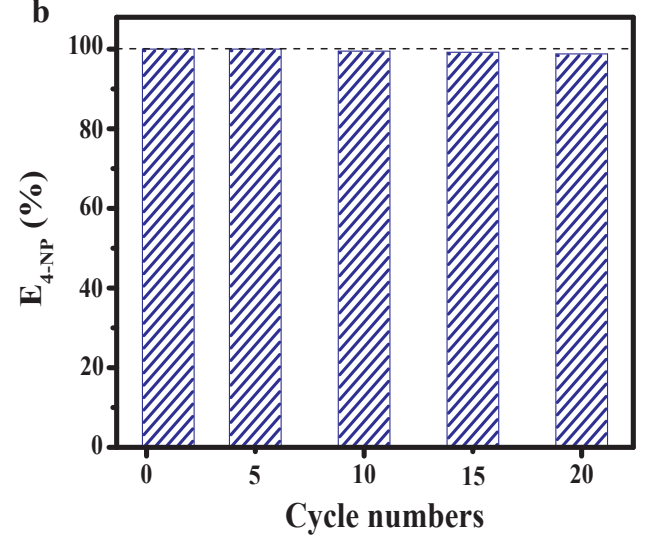

d

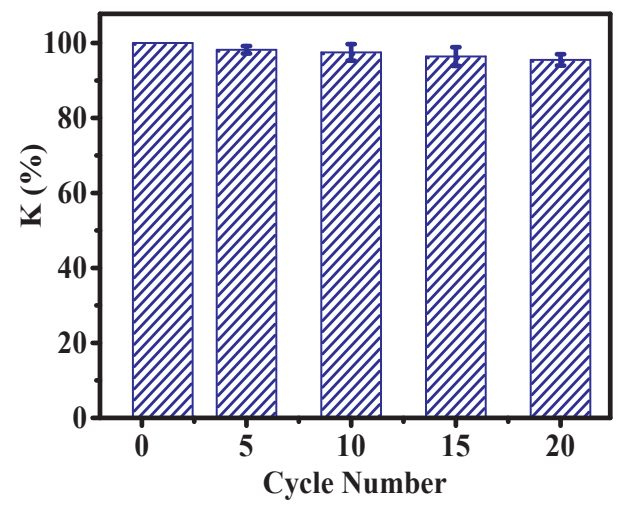

$\mathbf{f}$

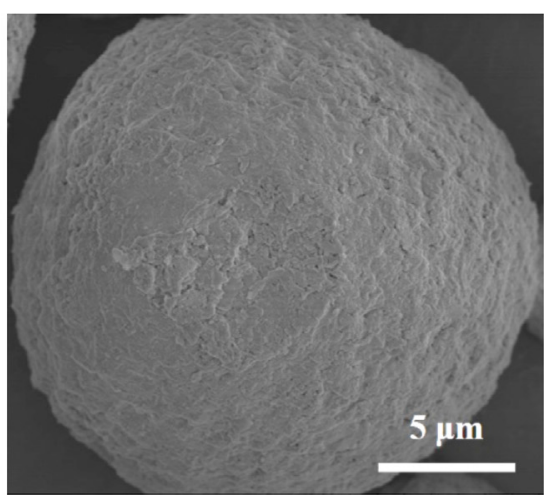

Fig. 6. The changes of apparent reduction rate constants $\mathrm{K}$ of microspheres in the reduction of MB (a) and 4-NP (b) with the prolonged cycle number. SEM images of SMt/MoS 2 microspheres after 20 cycles for MB (c) and 4-NP (d), respectively.

review \& editing. Fushuai Wang: Writing - original draft. Fengyan Xiao: Writing - original draft. Dawei Pan: Writing - review \& editing.

\section{Declaration of Competing Interest}

The authors declare that they have no known competing financial interests or personal relationships that could have appeared to influence the work reported in this paper.

\section{Acknowledgments}

The authors gratefully acknowledge the funding support from National Natural Science Foundation of China (No. 51703110 and 51903247), Key Science and Technology Program of Yantai City, China (No. 2018ZHGY075) and Shandong Key Laboratory of Coastal Environmental Processes, YICCAS (Grant No. 2019SDHADKFJJ15).
Appendix A. Supplementary material

Supplementary data to this article can be found online at https:// doi.org/10.1016/j.seppur.2020.117346.

\section{References}

[1] I. Ali, New generation adsorbents for water treatment, Chem. Rev. 112 (2012) 5073-5091.

[2] M. Bordbar, N. Negahdar, M. Nasrollahzadeh, Melissa Officinalis L. leaf extract assisted green synthesis of $\mathrm{CuO} / \mathrm{ZnO}$ nanocomposite for the reduction of 4-nitrophenol and Rhodamine B, Sep. Purif. Technol. 191 (2018) 295-300.

[3] Y. Wang, G.E. Chen, H.L. Wu, Z.L. Xu, J.J. Wan, L.J. Liu, S.J. Xu, Y.F. Kong, Q. Wu, J. Min, H.F. Mao, Fabrication of GO-Ag/PVDF/F127 modified membrane IPA coagulation bath for catalytic reduction of 4-nitrophenol, Sep. Purif. Technol. 235 (2020) 116143.

[4] Y.X. Wang, S. Ma, M.N. Huang, H. Yang, Z.L. Xu, Z. Xu, Ag NPs coated PVDF@TiO nanofiber membrane prepared by epitaxial growth on $\mathrm{TiO}_{2}$ inter-layer for 4-NP reduction application, Sep. Purif. Technol. 227 (2019) 115700.

[5] M. Zubair, M. Daud, G. McKay, F. Shehzad, M.A. Al-Harthi, Recent progress in layered double hydroxides (LDH)-containing hybrids as adsorbents for water 
remediation, Appl. Clay Sci. 143 (2017) 279-292.

[6] V. Vimonses, S. Lei, B. Jin, C.W.K. Chow, C. Saint, Adsorption of congo red by three Australian kaolins, Appl. Clay Sci. 43 (2009) 465-472.

[7] C.S. Patil, A.N. Kadam, D.B. Gunjal, V.M. Naik, S.W. Lee, G.B. Kolekar, A.H. Gore, Sugarcane molasses derived carbon sheet@sea sand composite for direct removal of methylene blue from textile wastewater: Industrial wastewater remediation through sustainable, greener, and scalable methodology, Sep. Purif. Technol. 247 (2020) 116997.

[8] P. Cartagena, M.E. Kaddouri, V. Cases, A. Trapote, D. Prats, Reduction of emerging micropollutants, organic matter, nutrients and salinity from real wastewater by combined MBR-NF/RO treatment, Sep. Purif. Technol. 110 (2013) 132-143.

[9] W. Zhang, X. Wang, Y. Zhang, B. van Bochove, E. Mäkilä, J. Seppälä, W. Xu, S. Willför, C. Xu, Robust shape-retaining nanocellulose-based aerogels decorated with silver nanoparticles for fast continuous catalytic discoloration of organic dyes, Sep. Purif. Technol. 242 (2020) 116523.

[10] A. Hatamifard, M. Nasrollahzadeh, J. Lipkowski, Green synthesis of a natrolite zeolite/palladium nanocomposite and its application as a reusable catalyst for the reduction of organic dyes in a very short time, RSC Adv. 5 (2015) 91372-91381.

[11] M. Atarod, M. Nasrollahzadeh, S.M. Sajadi, Green synthesis of a Cu/reduced graphene oxide $/ \mathrm{Fe}_{3} \mathrm{O}_{4}$ nanocomposite using Euphorbia wallichii leaf extract and its application as a recyclable and heterogeneous catalyst for the reduction of 4-nitrophenol and rhodamine B, RSC Adv. 5 (2015) 91532-91543.

[12] M. Nasrollahzadeh, M. Sajjadi, M.R. Tahsili, High efficiency treatment of organic inorganic pollutants using recyclable magnetic N-heterocyclic copper(II) complex and its antimicrobial applications, Sep. Purif. Technol. 238 (2020) 116403.

[13] M. Sajjadi, N.Y. Baran, T. Baran, M. Nasrollahzadeh, M.R. Tahsili,

M. Shokouhimehr, Palladium nanoparticles stabilized on a novel Schiff base modified Unye bentonite: Highly stable, reusable and efficient nanocatalyst for treating wastewater contaminants and inactivating pathogenic microbes, Sep. Purif. Technol. 237 (2020) 116383.

[14] S.-M.-G. Yek, D. Azarifar, M. Nasrollahzadeh, M. Bagherzadeh, M. Shokouhimehr, Heterogenized $\mathrm{Cu}(\mathrm{II})$ complex of 5-aminotetrazole immobilized on graphene oxide nanosheets as an efficient catalyst for treating environmental contaminants, Sep. Purif. Technol. 247 (2020) 116952.

[15] Z. Zhang, D. Jiang, D. Li, M. He, M. Chen, Construction of $\mathrm{SnNb}_{2} \mathrm{O}_{6}$ nanosheet/g$\mathrm{C}_{3} \mathrm{~N}_{4}$ nanosheet two-dimensional heterostructures with improved photocatalytic activity: Synergistic effect and mechanism insight, Appl. Catal., B 183 (2016) 113-123.

[16] H. Zhang, Ultrathin two-dimensional nanomaterials, ACS Nano 9 (2015) 9451-9469.

[17] S. Kumar, V. Sharma, K. Bhattacharyya, V. Krishnan, N-doped ZnO-MoS 2 binary heterojunctions: the dual role of $2 \mathrm{D} \mathrm{MoS}_{2}$ in the enhancement of photostability and photocatalytic activity under visible light irradiation for tetracycline degradation, Mater. Chem. Front. 1 (2017) 1093-1106.

[18] L. Guardia, J.I. Paredes, J.M. Munuera, S. Villar-Rodil, J.M.D. Tascon, Chemically exfoliated $\mathrm{MoS}_{2}$ nanosheets as an efficient catalyst for reduction reactions in the aqueous phase, ACS Appl. Mater. Inter. 6 (2014) 21702-21710.

[19] V.O. Koroteev, L.G. Bulusheva, I.P. Asanov, E.V. Shlyakhova, D.V. Vyalikh, A.V. Okotrub, Charge transfer in the $\mathrm{MoS}_{2}$ /carbon nanotube composite, J. Phys. Chem. C 115 (2011) 21199-21204.

[20] S. Chakrabarty, A. Mukherjee, S. Basu, RGO- $\mathrm{MoS}_{2}$ supported $\mathrm{NiCo}_{2} \mathrm{O}_{4}$ catalyst toward solar water splitting and dye degradation, ACS Sustain. Chem. Eng. 6 (2018) 5238-5247.

[21] N. Meng, J. Cheng, Y. Zhou, W. Nie, P. Chen, Green synthesis of layered 1T-MoS $/$ reduced graphene oxide nanocomposite with excellent catalytic performances for 4nitrophenol reduction, Appl. Surf. Sci. 396 (2017) 310-318.

[22] A. Pal, T.K. Jana, T. Roy, A. Pradhan, K. Chatterjee, $\mathrm{MoS}_{2}-\mathrm{TiO}_{2}$ nanocomposite with excellent adsorption performance and high antibacterial activity, Chemistryselect 3 (2018) 81-90.
[23] P. Chen, S. Zeng, Y. Zhao, S. Kang, T. Zhang, S. Song, Synthesis of unique-morphological hollow microspheres of $\mathrm{MoS}_{2} @$ montmorillonite nanosheets for the enhancement of photocatalytic activity and cycle stability, J. Mater. Sci. Technol. 41 (2020) 88-97.

[24] W. Wang, T. Wen, H. Bai, Y. Zhao, J. Ni, L. Yang, L. Xia, S. Song, Adsorption toward $\mathrm{Cu}(\mathrm{II})$ and inhibitory effect on bacterial growth occurring on molybdenum disulfide-montmorillonite hydrogel surface, Chemosphere 248 (2020) 126025.

[25] L. Yang, Q. Wang, J.R. Rangel-Mendez, F. Jia, S. Song, B. Yang, Self-assembly montmorillonite nanosheets supported hierarchical $\mathrm{MoS}_{2}$ as enhanced catalyst toward methyl orange degradation, Mater. Chem. Phys. 246 (2020) 122829.

[26] E.D.A. Mário, C. Liu, C.I. Ezugwu, S. Mao, F. Jia, S. Song, Molybdenum disulfide/ montmorillonite composite as a highly efficient adsorbent for mercury removal from wastewater, Appl. Clay Sci. 184 (2020) 105370.

[27] K. Peng, H. Wang, H. Gao, P. Wan, M. Ma, X. Li, Emerging hierarchical ternary 2D nanocomposites constructed from montmorillonite, graphene and $\mathrm{MoS}_{2}$ for enhanced electrochemical hydrogen evolution, Chem. Eng. J. 124704 (2020).

[28] A. Joseph, K. Vellayan, B. González, M.A. Vicente, A. Gil, Effective degradation of methylene blue in aqueous solution using Pd-supported Cu-doped Ti-pillared montmorillonite catalyst, Appl. Clay Sci. 168 (2019) 7-10.

[29] Q. Zhang, Z. Yan, J. Ouyang, Y. Zhang, H. Yang, D. Chen, Chemically modified kaolinite nanolayers for the removal of organic pollutants, Appl. Clay Sci. 157 (2018) 283-290.

[30] K. Peng, L. Fu, H. Yang, J. Ouyang, A. Tang, Hierarchical MoS2 intercalated clay hybrid nanosheets with enhanced catalytic activity, NANO Res. 10 (2017) 570-583.

[31] K. Peng, J. Wang, H. Wang, X. Li, P. Wan, H. Zhang, L. Bai, MoS2 nanosheets supported on carbon hybridized montmorillonite as an efficient heterogeneous catalyst in aqueous phase, Appl. Clay Sci. 183 (2019) 105346.

[32] A. Stunda-Zujeva, Z. Irbe, L. Berzina-Cimdina, Controlling the morphology of ceramic and composite powders obtained via spray drying-A review, Ceram. Int. 43 (2017) 11543-11551.

[33] Y. Qin, N. Wang, Y. Zhou, Y. Huang, H. Niu, J.Y. Dong, Fabrication of nanofillers into a granular "Nanosupport" for Ziegler-Natta catalysts: Towards scalable in situ preparation of polyolefin nanocomposites, Macromol. Rapid Commun. 32 (2011) 1052-1059.

[34] N. Wang, F. Xiao, J. Zhang, H. Zhou, Y. Qin, D. Pan, Spherical montmorillonitesupported nano-silver as a self-sedimentary catalyst for methylene blue removal, Appl. Clay Sci. 174 (2019) 146-151.

[35] F. Xiao, H. Ren, H. Zhou, H. Wang, N. Wang, D. Pan, Porous montmorillonite@ graphene oxide@Au nanoparticle composite microspheres for organic dye degradation, ACS Appl. Nano Mater. 2 (2019) 5420-5429.

[36] F. Xiao, Y. Qin, N. Wang, D. Pan, Towards mass production of Au nanoparticles supported on montmorillonite microspheres for catalytic reduction of 4-nitrophenol, Appl. Clay Sci. 166 (2018) 74-79.

[37] N. Wang, Z. Zhang, J. Huang, Y. Hu, Facile synthesis of copper ions chelated sand via dopamine chemistry for recyclable and sustainable catalysis, Chem. Eng. Sci. 203 (2019) 312-320.

[38] Y. Liu, K. Ai, L. Lu, Polydopamine and its derivative materials: synthesis and promising applications in energy, environmental, and biomedical fields, Chem. Rev. 114 (2014) 5057-5115.

[39] N. Wang, Y. Hu, Z. Zhang, Sustainable catalytic properties of silver nanoparticles supported montmorillonite for highly efficient recyclable reduction of methylene blue, Appl. Clay Sci. 150 (2017) 47-55.

[40] W.P. Gonçalves, V.J. Silva, R.R. Menezes, G.A. Neves, H.L. Lira, L.N.L. Santana, Microstructural, physical and mechanical behavior of pastes containing clays and alumina waste, Appl. Clay Sci. 137 (2017) 259-265.

[41] Y. Tan, K. Yu, T. Yang, Q. Zhang, W. Cong, H. Yin, Z. Zhang, Y. Chen, Z. Zhu, The combinations of hollow MoS2 micro@nano-spheres: one-step synthesis, excellent photocatalytic and humidity sensing properties, J. Mater. Chem. C 2 (2014) 5422-5430. 